NUC-MINN-95/20-T

\title{
Role of Hyperon Negative Energy Sea in Nuclear Matter
}

\author{
P.J. Ellis and S.B. Parendo \\ School of Physics and Astronomy, University of Minnesota, \\ Minneapolis, MN 55455 \\ M. Prakash \\ Physics Department, SUNY at Stony Brook, \\ Stony Brook, NY 11794
}

\begin{abstract}
We have examined the contribution of the filled negative energy sea of hyperons to the energy/particle in nuclear matter at the one and two loop levels. While this has the potential to be significant, we find a strong cancellation between the one and two loop contributions for our chosen parameters so that hyperon effects can be justifiably neglected.
\end{abstract}


In the vacuuum Dirac particles have a filled negative energy sea with the positive energy states empty. Zero-point fluctuations give rise to the vacuum energy. In matter, the presence of positive energy particles results in a modification of the vacuum energy due to interactions. At the hadronic level, this implies that, even though only nucleon positive energy states are filled in nuclear matter (provided the density is not too high), the negative energy seas of particles other than nucleons can play a role. The purpose of this paper to investigate the situation for the hyperons, which, together with the nucleons, make up the basic octet. Since the hyperon coupling constants are generally not well known, our aim is to obtain a first semi-quantitative estimate to ascertain whether significant uncertainty is introduced into the description of simple equilibrium nuclear matter. We shall carry out our study for the one loop (Hartree) and two loop (Fock) diagrams of Fig. 1, since, intuitively, one might expect some cancellation between them, although as we shall see this is by no means always the case.

We shall employ the Walecka Lagrangian, which has been widely used in relativistic calculations of nuclear matter and finite nuclei [1]. Here the baryonic interactions are generated by the exchange of scalar $\sigma$ and vector $\omega$ mesons. The Lagrangian takes the form

$$
\begin{aligned}
\mathcal{L}= & \sum_{B} \bar{B}\left(i \gamma^{\mu} \partial_{\mu}-g_{\omega B} \gamma^{\mu} \omega_{\mu}-M_{B}+g_{\sigma B} \sigma\right) B \\
& -\frac{1}{4} \omega_{\mu \nu} \omega^{\mu \nu}+\frac{1}{2} m_{\omega}^{2} \omega_{\mu} \omega^{m u}+\frac{1}{2} \partial_{\mu} \sigma \partial^{\mu} \sigma-\frac{1}{2} m_{\sigma}^{2} \sigma^{2}
\end{aligned}
$$

where $M_{B}$ is the vacuum baryon mass and the field strength tensor is $\omega_{\mu \nu}=$ $\partial_{\mu} \omega_{\nu}-\partial_{\nu} \omega_{\mu}$. The baryons $B$ included in the sum are nucleons, $n, p$, and hyperons, $\Lambda, \Sigma^{+}, \Sigma^{-}, \Sigma^{0}, \Xi^{-}$and $\Xi^{0}$. 
For purely nucleon degrees of freedom many calculations have been carried out in the relativistic Hartree approximation which includes the one loop diagram of Fig. 1(a). More recently the exchange or Fock contribution, represented by the two loop diagram of Fig. 1(b), has been examined. The first calculation was carried out by Furnstahl, Perry and Serot [2] and the diagram was found to be very large. Subsequently it was pointed out that the composite nature of nucleons suggests that form factors should be included at the vertices; these are represented by the heavy dots in Fig. 1(b). Using phenomenological monopole form factors the two loop contribution was very substantially reduced [3] so that it was much smaller than the one loop result. Recently progress has been made in attempting to calculate the form factors by allowing for the exchange of soft vector mesons at the vertex [四]. The results are quite similar to those obtained with phenomenological form factors.

Allowing for the presence of hyperons, the one loop Hartree expression for the sigma field is

$$
m_{\sigma}^{2} \sigma=\frac{2 g_{\sigma N}}{\pi^{2}} \int_{0}^{k_{f}} d k k^{2} \frac{M_{N}^{*}}{\sqrt{k^{2}+M_{N}^{* 2}}}+\sum_{B} g_{\sigma B} \frac{\partial \Delta \mathcal{E}\left(M_{B}^{*}, M_{B}\right)}{\partial M_{B}^{*}},
$$

where $N$ denotes a nucleon and the baryon effective masses are $M_{B}^{*}=M_{B}-$ $g_{\sigma B} \sigma$. The density is related to the Fermi momentum by the usual expression $n=2 k_{f}^{3} /\left(3 \pi^{2}\right)$. For the renormalization needed for the negative energy sea we use the standard Chin-Walecka [5] prescription, so that

$$
\begin{array}{r}
\Delta \mathcal{E}\left(M^{*}, M\right)=-\frac{1}{8 \pi^{2}}\left[M^{* 4} \ln \frac{M^{*}}{M}+M^{3}\left(M-M^{*}\right)-\frac{7}{2} M^{2}\left(M-M^{*}\right)^{2}\right. \\
\left.+\frac{13}{3} M\left(M-M^{*}\right)^{3}-\frac{25}{12}\left(M-M^{*}\right)^{4}\right] .
\end{array}
$$


The values of the nucleon parameters used are $g_{\sigma N}^{2}=54.3, m_{\sigma}=458 \mathrm{MeV}$, $g_{\omega N}^{2}=102.8$ and $m_{\omega}=783 \mathrm{MeV}$, which saturate nuclear matter (without hyperons) at $k_{f}=1.3 \mathrm{fm}^{-1}$ with a binding energy of $15.75 \mathrm{MeV}$ [2]. The contribution to the energy density from a given hyperon species at the one loop level is then $\Delta E\left(M_{H}^{*}, M_{H}\right)$ for which we need to specify the hyperon coupling constants.

The two-loop contributions we treat perturbatively since they are small when form factors are employed [3, 国]. Formally, using point vertices Fig. 1(b) gives a contribution to the energy density

$$
\mathcal{E}_{p t}=\frac{1}{2} \int \frac{d^{4} p_{1}}{(2 \pi)^{4}} \frac{d^{4} p_{2}}{(2 \pi)^{4}} \operatorname{Tr}\left[S\left(p_{1}\right) \Gamma_{1} S\left(p_{2}\right) \Gamma_{2}\right] D(k),
$$

where $S(D)$ represent baryon (meson) propagators, $\Gamma$ represents a point vertex, $k=p_{1}-p_{2}$ and Lorentz indices have been suppressed. For hyperons, the two loop contribution is due to vacuum fluctuations and we use the expression given in Ref. [2]. We allow for the composite nature of hyperons by introducing form factors. At each vertex we employ a phenomenological monopole form, $\left(1-q^{2} / \Lambda_{\text {cut }}^{2}\right)^{-1}$, where $q$ is the four-momentum transfer to the meson $\left(q^{2}<0\right)$ and $\Lambda_{\text {cut }}$ is a cut-off parameter; alternatively this could be viewed as a single dipole form factor at one of the vertices. The energy density can be written

$$
\mathcal{E}=\frac{\Lambda_{\text {cut }}^{2}}{\Lambda_{\text {cut }}^{2}-m^{2}}\left[\frac{\Lambda_{\text {cut }}^{2}}{\Lambda_{\text {cut }}^{2}-m^{2}}\left(\mathcal{E}_{p t}\left(m^{2}\right)-\mathcal{E}_{p t}\left(\Lambda_{\text {cut }}^{2}\right)\right)+\frac{d \mathcal{E}_{p t}\left(\Lambda_{\text {cut }}^{2}\right)}{d \ln \Lambda_{\text {cut }}^{2}}\right],
$$

where $m$ is the mass of the exchanged meson.

Since the hyperon parameters which enter are poorly known, it is well to begin with a simple exploration of parameter space. Thus we focus on a single 
hypothetical hyperon and consider a vacuum mass of 1 or $2 \mathrm{GeV}$. We choose the ratios of the hyperon to nucleon coupling constants, $x_{\sigma H}=g_{\sigma H} / g_{\sigma N}$ and $x_{\omega H}=g_{\omega H} / g_{\omega N}$, to both be $\frac{2}{3}$, as suggested by simple quark counting arguments. The contribution to the energy/particle, i.e. $\mathcal{E} / n$, of nuclear matter is shown in Table 1. Results are given for values of the nucleon Fermi momentum of $k_{f}=1.3,1.6$ and $1.9 \mathrm{fm}^{-1}$, corresponding to densities of $n_{0}$, $1.9 n_{0}$ and $3.1 n_{0}$, respectively, where equilibrium nuclear matter density is denoted by $n_{0}$. These densities should be below the threshold for positive energy hyperons. Consider first the cases where $\Lambda_{\text {cut }}=\infty$, i.e. the form factor is simply unity. The leading contribution to the energy/particle for both the one and two loop results is proportional to $\left(M_{H}^{*}-M_{H}\right)^{5} / M_{H} k_{f}^{3}$. This accounts quite well for the relative magnitudes of the results, although when comparing different hyperon masses at the two loop level it must be borne in mind that there is a residual dependence on the ratio of the meson mass to the hyperon mass which results in a $12 \%$ deviation from this simple formula. The negative two loop contribution from $\omega$-exchange dominates so that the overall contribution from the negative energy sea is negative, as with nucleons, but the magnitude is smaller here since the effective mass is larger. When we introduce form factors with $\Lambda_{\text {cut }}=2 \mathrm{GeV}$ the dominant feature is that the $\omega$ contribution is reduced by a larger amount than the $\sigma$ contribution- by a factor of $\sim 2$ (5) for $M_{H}=1$ (2) GeV. This is sufficient for the net two-loop result to be positive for $M_{H}=2 \mathrm{GeV}$. The case $\Lambda_{\text {cut }}=$ $1 \mathrm{GeV}$ is more dramatic in that for $M_{H}=1 \mathrm{GeV}$ the two-loop $\sigma$ and $\omega$ contributions essentially cancel, while for $M_{H}=2 \mathrm{GeV}$ the $\omega$ contribution becomes positive. For a given $M_{H}$ and $\Lambda_{\text {cut }}$ the relative values at different 
values of $k_{f}$ still follow the leading order behavior mentioned above. We see that the two loop results are sensitive to the hyperon mass and give a net positive result for the larger mass, assuming $\Lambda_{\text {cut }}$ is the same for both mesons, and this would slightly increase the one loop contribution.

We turn now to the effect of the filled negative energy sea of $\Lambda, \Sigma$ and $\Xi$ hyperons upon the energy of nuclear matter. Following Glendenning and Moszkowski [6], we constrain the coupling constants of the $\Lambda$-hyperon by requiring that the correct energy be obtained for the lowest $\Lambda$ level in nuclear matter at saturation. Defining, as before, the ratio $x_{\sigma \Lambda}=g_{\sigma \Lambda /} g_{\sigma N}$ etc., this gives at the Hartree level

$$
-28=x_{\omega \Lambda} g_{\omega N} \omega_{0}-x_{\sigma \Lambda} g_{\sigma N} \sigma,
$$

in units of MeV. Choosing $x_{\omega \Lambda}=\frac{2}{3}$ on the basis of quark counting arguments, we find $x_{\sigma \Lambda}=0.614$ and these values yield reasonable properties for neutron stars according to Ref. [6]. For the $\Sigma$ we rely on a recent analysis of $\Sigma^{-}$ atomic data by Mareš et al [7]. For consistency we pick their case with $x_{\omega \Sigma}=\frac{2}{3}$ which yields a good fit with $x_{\sigma \Sigma}=0.54$. As regards the $\Xi$, in the absence of better information we take the simple quark model estimate $x_{\omega \Xi}=x_{\sigma \Xi}=\frac{1}{3}$. Using the analogue of Eq. (6), the energy of the lowest $\Sigma$ and $\Xi$ levels in equilibrium nuclear matter are -10 and $-21 \mathrm{MeV}$ respectively. The latter is reasonable according to the discussion of Schaffner et al. [8] . As regards the $\Sigma$ energy, Mareš et al [7] suggest that the optical potential is repulsive in the nuclear interior. A modest adjustment of $x_{\sigma \Sigma}$ to yield zero or a small positive energy would not qualitatively change our results. Finally we need to specify the form factors. The Jülich fit to hyperon-nucleon 
scattering [9] indicates that, unlike nucleons, hyperons favor a larger cut-off for $\omega$ exchange, $\Lambda_{\text {cut }}=2 \mathrm{GeV}$, than for $\sigma$ exchange, $\Lambda_{\text {cut }}=1 \mathrm{GeV}$. The cut-off there was in the 3-momentum, but we shall simply adopt these values as reasonable estimates.

The effect of the hyperons upon the self-consistent $\sigma$ field equation (2) is slight. It results in an $\sim 1 \%$ reduction in the field which does not significantly affect the nuclear matter saturation properties obtained with nucleons alone. The hyperon contributions to the energy/particle, with the parameters discussed above, are shown in Table 2. The $\Xi$ is less important than the other hyperons due to its smaller coupling and resulting larger effective mass. The total hyperon one-loop contribution amounts to 3-4\% of the pure nucleon energy/particle at $k_{f}=1.3$ and 1.9. This is modest, but not negligible. The two-loop contribution is dominated by $\omega$ exchange so that for each hyperon species the net result is negative. At all three values of $k_{f}$, the total hyperon results at the one and two loop levels show a strong cancellation so that the net hyperon contribution is negligible. Note that this depends on using a larger $\Lambda_{\text {cut }}$ for the $\omega$ which gives a sizeable, negative two-loop result ( $c f$. Table 1). We should also comment briefly on uncertainties in the coupling constants. Consider the $\Sigma$ : Mareš et al [7] report that their best fits are obtained with $x_{\omega \Sigma}$ in the range $\frac{2}{3}$ to 1 . With $x_{\omega \Sigma}=1$ they obtain $x_{\sigma \Sigma}=0.77$ which, with our parameters, would give the lowest $\Sigma$ level in nuclear matter at $-4 \mathrm{MeV}$. Since the sigma coupling is larger here the effective mass is smaller which leads to enhanced contributions, for example at saturation the one plus two loop contribution is $1.6-3.0=-1.4 \mathrm{MeV}$, by no means negligible. Also the Jülich group [9] have suggested a large value 
of $x_{\sigma \Sigma}=1.28$, while $x_{\omega \Sigma}$ remains $\frac{2}{3}$. This yields a large value for the net one plus two loop contribution of $14.5 \mathrm{MeV}$, but this should not be taken seriously since these parameters yield an unreasonably large binding for the lowest $\Sigma$ level in nuclear matter.

Our aim has been to investigate the contribution of the filled negative energy sea of hyperons to the binding energy of nuclear matter. With the most reasonable parameters that we could find there was a strong degree of cancellation between the one and two loop contributions so that the sum was negligibly small. This is very satisfactory since it implies that such effects can be safely neglected, as has been common practice. This conclusion is, however, strongly dependent on the rather uncertain values of the parameters and further efforts to tie them down would be very valuable.

This work was supported in part by the U. S. Department of Energy under grant numbers DE-FG02-87ER40328 and DE-FG02-88ER40388.

\section{References}

[1] B.D. Serot and J.D. Walecka, Advance in Nuclear Physics, Vol. 16, ed. J.W. Negele and E. Vogt (Plenum, NY, 1986); B.D. Serot, Rep. Prog. Phys. 55, 1855 (1992).

[2] R.J Furnstahl, R.J. Perry and B.D. Serot, Phys. Rev. C40 (1989) 321.

[3] M. Prakash, P.J. Ellis and J.I. Kapusta, Phys. Rev. C45 (1992) 2518; R. Friedrich, K. Wehrberger and F. Beck, Phys. Rev. C46 (1992) 188. 
[4] M.P. Allendes and B.D. Serot, Phys. Rev. C45 (1992) 2975; B.D. Serot and H. Tang, Phys. Rev. C51 (1995) 969.

[5] S.A. Chin, Ann. Phys. (NY) 108 (1977) 301.

[6] N. K. Glendenning and S.A. Moszkowski, Phys. Rev. Lett. 67 (1991) 2414.

[7] J. Mareš, E. Friedman, A. Gal and B.K. Jennings, preprint (1995) nuclth/9505003.

[8] J. Schaffner, C.B. Dover, A. Gal, C. Greiner, D.J. Millener and H. Stöcker, Ann. Phys. (NY) 235 (1994) 35.

[9] A. Reuber, K. Holinde and J. Speth, Nucl. Phys. A570 (1994) 543. 
Table 1. Contribution of a single hypothetical hyperon, of mass 1 or $2 \mathrm{GeV}$, to the energy/particle at the one and two loop levels. Results are given for several values of the Fermi momentum, $k_{f}$, and the form factor cut-off parameter, $\Lambda_{\text {cut }}$.

\begin{tabular}{|c|c|c|c|c|c|c|c|}
\hline \multirow{3}{*}{$\begin{array}{c}k_{f} \\
\mathrm{fm}^{-1}\end{array}$} & \multirow{3}{*}{$\begin{array}{c}\text { Mass } M_{H} \\
\mathrm{GeV}\end{array}$} & \multirow{3}{*}{$M_{H}^{*} / M_{H}$} & \multirow{3}{*}{$\begin{array}{c}\text { One Loop } \\
\mathrm{MeV}\end{array}$} & \multicolumn{4}{|c|}{ Two Loop } \\
\hline & & & & Cut-off $\Lambda_{\text {cut }}$ & $\sigma$ & $\omega$ & Total \\
\hline & & & & $\mathrm{GeV}$ & $\mathrm{MeV}$ & $\mathrm{MeV}$ & $\mathrm{MeV}$ \\
\hline \multirow{6}{*}{1.3} & \multirow{3}{*}{1} & \multirow{3}{*}{0.83} & \multirow{3}{*}{0.307} & $\infty$ & 0.397 & -1.555 & -1.158 \\
\hline & & & & 2 & 0.199 & -0.393 & -0.194 \\
\hline & & & & 1 & 0.068 & -0.067 & 0.001 \\
\hline & \multirow{3}{*}{2} & \multirow{3}{*}{0.92} & \multirow{3}{*}{0.153} & $\infty$ & 0.227 & -0.881 & -0.655 \\
\hline & & & & 2 & 0.041 & -0.030 & 0.010 \\
\hline & & & & 1 & 0.008 & 0.005 & 0.013 \\
\hline \multirow{6}{*}{1.6} & \multirow{3}{*}{1} & \multirow{3}{*}{0.76} & \multirow{3}{*}{1.058} & $\infty$ & 1.335 & -5.234 & -3.899 \\
\hline & & & & 2 & 0.682 & -1.360 & -0.678 \\
\hline & & & & 1 & 0.235 & -0.233 & 0.001 \\
\hline & \multirow{3}{*}{2} & \multirow{3}{*}{0.88} & \multirow{3}{*}{0.529} & $\infty$ & 0.775 & -3.013 & -2.239 \\
\hline & & & & 2 & 0.141 & -0.105 & 0.036 \\
\hline & & & & 1 & 0.029 & 0.016 & 0.045 \\
\hline \multirow{6}{*}{1.9} & \multirow{3}{*}{1} & \multirow{3}{*}{0.70} & \multirow{3}{*}{1.866} & $\infty$ & 2.307 & -9.047 & -6.741 \\
\hline & & & & 2 & 1.198 & -2.405 & -1.207 \\
\hline & & & & 1 & 0.413 & -0.415 & -0.001 \\
\hline & \multirow{3}{*}{2} & \multirow{3}{*}{0.85} & \multirow{3}{*}{0.930} & $\infty$ & 1.352 & -5.260 & -3.908 \\
\hline & & & & 2 & 0.248 & -0.185 & 0.062 \\
\hline & & & & 1 & 0.050 & 0.029 & 0.079 \\
\hline
\end{tabular}


Table 2. Contribution of the $\Lambda, \Sigma$ and $\Xi$ hyperons to the energy/particle at the one and two loop levels for three values of the Fermi momentun, $k_{f}$. The parameterization is discussed in the text.

\begin{tabular}{|ccc|c|ccc|}
\hline \hline & & & & \multicolumn{3}{|c|}{ Two Loop } \\
$k_{f}$ & Particle & $M_{B}^{*} / M_{B}$ & One Loop & $\sigma$ & $\omega$ & Total \\
$\mathrm{fm}^{-1}$ & & & $\mathrm{MeV}$ & $\mathrm{MeV}$ & $\mathrm{MeV}$ & $\mathrm{MeV}$ \\
\hline \multirow{2}{*}{1.3} & $\Lambda$ & 0.86 & 0.179 & 0.028 & -0.188 & -0.160 \\
& $\Sigma$ & 0.89 & 0.273 & 0.030 & -0.251 & -0.221 \\
& $\Xi$ & 0.94 & 0.014 & 0.000 & -0.003 & -0.002 \\
\hline Totals & & & 0.466 & & & -0.383 \\
\hline & $\Lambda$ & 0.80 & 0.609 & 0.096 & -0.643 & -0.547 \\
1.6 & $\Sigma$ & 0.83 & 0.927 & 0.103 & -0.856 & -0.754 \\
& $\Xi$ & 0.91 & 0.048 & 0.002 & -0.009 & -0.007 \\
\hline Totals & & & 1.584 & & & -1.308 \\
\hline & $\Lambda$ & 0.75 & 1.068 & 0.169 & -1.132 & -0.963 \\
1.9 & $\Sigma$ & 0.79 & 1.623 & 0.180 & -1.505 & -1.325 \\
& $\Xi$ & 0.89 & 0.083 & 0.003 & -0.015 & -0.013 \\
\hline Totals & & & 2.774 & & & -2.301 \\
\hline \hline
\end{tabular}




\section{Figure Caption}

Fig. 1. (a) One loop Hartree and (b) two loop Fock diagram; the full (dashed) lines represent baryons (mesons) and the heavy dots indicate form factors. 




(a)

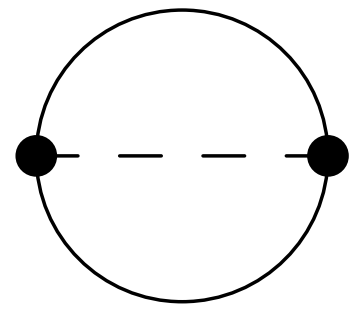

(b) 